\title{
Palpable Masses Numeric Result in
}

\section{Standard Unit}

National Cancer Institute

\section{Source}

National Cancer Institute. Palpable Masses Numeric Result in Standard Unit. NCI

Thesaurus. Code C119918.

The numerical identifier of a palpable masses assessment result in standard units. 